\section{RE: INTERPAPILLARY \\ MUSCLE DISTANCE \\ INDEPENDENTLY AFFECTS \\ SEVERITY OF FUNCTIONAL \\ MITRAL REGURGITATION IN \\ PATIENTS WITH SYSTOLIC \\ LEFT VENTRICULAR \\ DYSFUNCTION \\ To the Editor:}

The recent article by Kim and coauthors $^{1}$ adds to the steadily growing body of evidence that identifies papillary separation as a dominant factor in the mitral insufficiency that is caused by left ventricular dysfunction. It does not, however, explain why this is so. I performed an old fashioned gross anatomic study, which revealed the mechanism. ${ }^{2}$

The normal movements of the mitral valve are produced by changing fluid forces interacting with the leaflets, which are subject to the control of the chordae. In a normal heart, the changes in the annulus, ventricular wall, and papillary muscles are synchronized with the fluid forces. Leaflet flexibility, such that the leaflet tissue bends under its own weight, is essential to this behavior.

The critical gross anatomy that governs the behavior of the leaflets in the presence of papillary displacement is the fact that the distance from the origin of a second-order, load-bearing, main chorda to its insertion into the junction of the

\footnotetext{
The Editor welcomes submissions for possible publication in the Letters to the Editor section that consist of commentary on an article published in the Journal or other relevant issues. Authors should: - Include no more than 500 words of text, three authors, and five references. - Type with double-spacing - See http://jtcs.ctsnetjournals.org/misc/ifora.shtm for detailed submission instructions. - Submit the letter electronically via jtcvs.editorialmanager.com. Letters commenting on an article published in the JTCVS will be considered if they are received within 6 weeks of the time the article was published. Authors of the article being commented on will be given an opportunity of offer a timely response ( 2 weeks) to the letter. Authors of letters will be notified that the letter has been received. Unpublished letters cannot be returned.
}

meeting of rough and smooth zones of the anterior leaflet is less than the combined lengths of the origin of a first-order, aligning chorda to its free-edge insertion and the length of the rough zone from free edge to the insertion of the main chorda.

In their study, Kim and coauthors ${ }^{1}$ discuss the classic tenting appearance of the closed mitral valve in the presence of papillary displacement. They emphasize that papillary separation has a greater effect on mitral insufficiency than either apical or posterior papillary displacement.

These consequences of papillary separation are explained by in vitro studies of the effects of papillary position on mitral valves with a rigid tight reduction of the mural annulus. ${ }^{2}$

With apical displacement of papillary chordal origins, the pull of a main chordal insertion is transmitted to the leaflet tissue between the chordal insertion and the annulus. This stiffens and restricts the mobility of the anterior leaflet from the annulus to the rough zone. This stiffened part of the anterior leaflet and a less obvious similar stiffened portion of the posterior central scallop together produce the appearance of a tent while the still flexible rough zone of the mitral leaflets is capable of at least partial closure. When the papillary muscles are separated, however, this also tightens the central first-order chordae. With these chordae tight, the rough zone between the free edge and the insertion of the main chordae also becomes stiff. The whole central portion of the anterior leaflet and the central scallop from base to free edge have at this point lost the flexibility essential to normal opening and closing. Further narrowing of the annuloplasty increases the tightness of the chordae and the stiffness of the leaflets.

Robert W. M. Frater, MBChB, MSSurg, FRCS, Professor Emeritus Department of Cardiothoracic Surgery

\section{Albert Einstein College of Medicine Montefiore Medical Center}

Bronx, NY

\section{References}

1. Kim K, Kaji S, An Y, Nishino T, Tani T, Kitai T, et al. Interpapillary muscle distance independently affects severity of functional mitral regurgitation in patients with systolic left ventricula dysfunction. J Thorac Cardiovasc Surg. 2014 148:434-40.e1.

2. Frater RW. The effects on cordal and leaflet stiffness of severe apical, posterior, and outward papillary displacement in advanced ventricular mechanism heart failure and mitral insufficiency. J Heart Valve Dis. 2011;20:608-18.

3. He S, Weston MW, Lemmon J, Jensen M, Levine RA, Yoganathan AJ. Geometric distribution of chordae tendineae: an important feature in mitral valve function. J Heart Valve Dis. 2000;9:492-501; discussion 502-3.

http://dx.doi.org/10.1016/ j.jtcvs.2014.08.044

\section{WHY DOES PAPILLARY SEPARATION PLAY A MAJOR ROLE IN FUNCTIONAL MITRAL REGURGITATION CAUSED BY LEFT VENTRICULAR FUNCTION?}

\section{Reply to the Editor:}

Functional mitral regurgitation (FMR) is a common complication in patients with ischemic heart disease, and it occurs as a consequence of left ventricular dysfunction. Previous studies have shown that leaflet tethering by displaced papillary muscles is an essential pathway of FMR. ${ }^{1}$ In addition, the tethering distance of papillary muscles between papillary muscle tips and the anterior annulus is a dominant factor in the severity of FMR. ${ }^{2}$ Reducing the distance between the anterior and posterior papillary muscles, the interpapillary muscle distance (IPMD), with the papillary muscle approximation technique is effective for restoring leaflet coaptation. Papillary separation is therefore considered a dominant factor in FMR caused by left ventricular dysfunction. The reason that papillary separation plays an important role in 


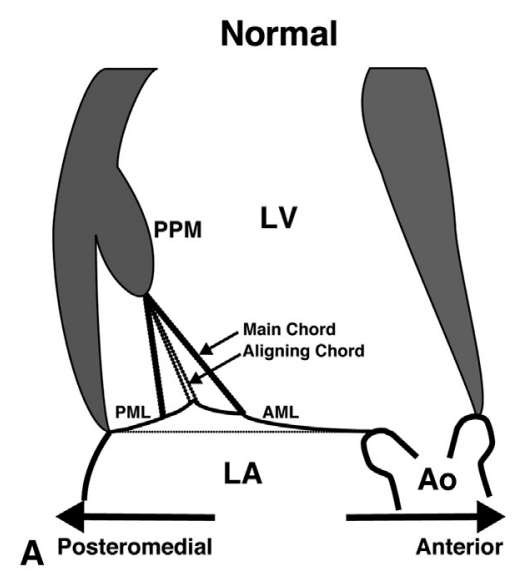

Normal

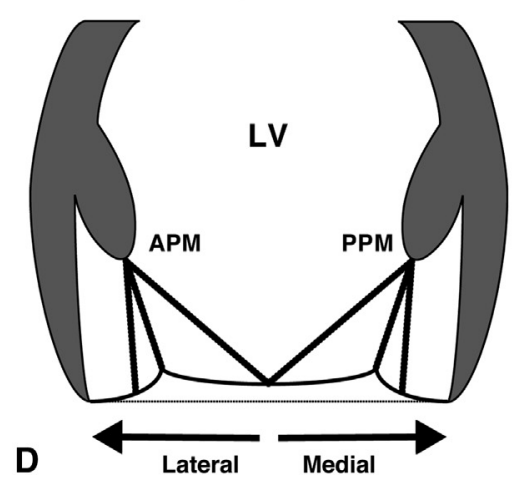

Effects of Apical Shift

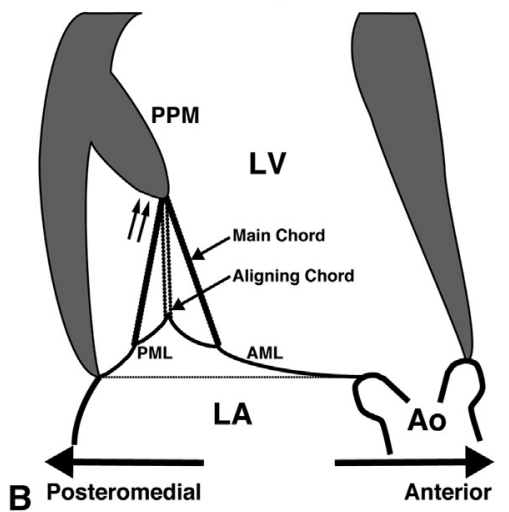

Effects of Apical Shift

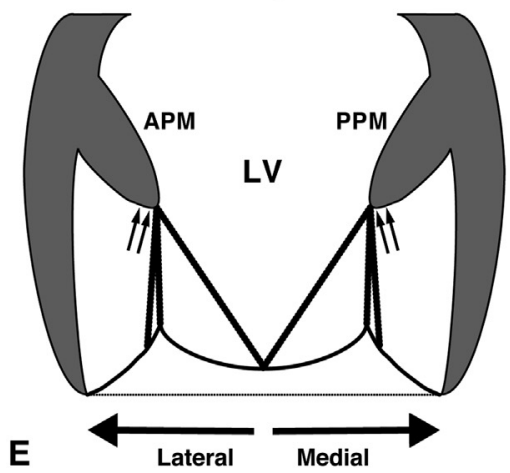

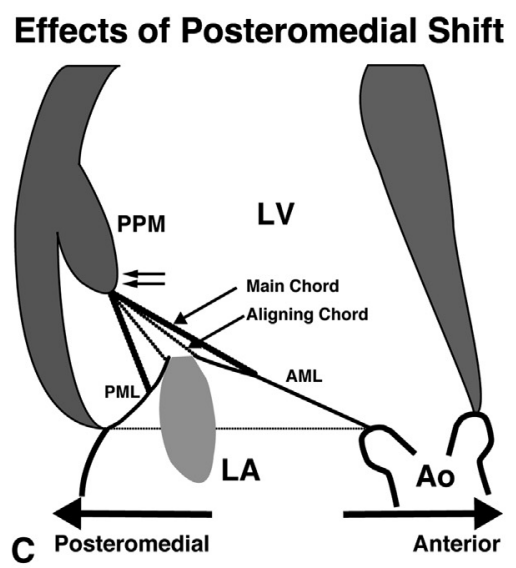

FIGURE 1. A-C, Schema shows a cross-sectional image of the mitral valve in the anterior-posteromedial direction. A, Control. B, Apical displacement of papillary muscle lifts up whole leaflets, except for the rough zone, which allows at least partial closure. C, Mediolateral displacement of papillary muscle lifts up the rough zone of the anterior and posterior leaflets, which leads to insufficient mitral closure. D-F, Schema shows a cross-sectional image of the mitral valve in the mediolateral (commissure-commissure) direction. D, Control. E, Apical displacement of both papillary muscles does not lift up the central portion of both leaflets. F, Mediolateral displacement of the anterior and posterior papillary muscles lifts up the central portion of leaflets and increases the tethering of whole leaflets. $P P M$, Posterior papillary muscle; $L V$, left ventricle; $P M L$, posterior mitral leaflet; $A M L$, anterior mitral leaflet; $L A$, left atrium; $A o$, aorta; $A P M$, anterior papillary muscle.

the mechanism of FMR, however, is unclear.

In his letter, Frater clearly illustrates why papillary separation is important in this mechanism. He observes that papillary muscle separation with nonapical outward (posterolateral or posteromedial) displacement causes greater impairment of systolic mitral closure than does apical displacement (Figure 1, $A-C)$. Notably, tension in a main chord (second-order chord) is 3 -fold that of an aligning chord (first-order chord) inserted into the free edge. ${ }^{4}$ With apical displacement of the papillary muscle, the leaflet tissue between main chordal insertion and the annulus is tethered, which restricts the mobility of the anterior leaflet from the annulus to the rough zone, leaving the aligning chord loose. This tethered anterior leaflet and similarly tethered posterior scallop consists of leaflet tenting, while the still flexible rough zone of the leaflets allows at least partial closure (Figure 1, B). In contrast, when the papillary muscles are displaced in the posteromedial or posterolateral direction, leading to papillary muscle separation, the main and aligning chords are tightened. Consequently, the whole central portion of the anterior leaflet and posterior middle scallop, including the rough zone of the leaflets, loses the flexibility necessary for leaflet closure (Figure 1, C).
Frater explains the geometric relationship between papillary muscle and leaflets in an anteriorto-posteromedial or anterior-toposterolateral direction. There might be another effect on leaflet tethering of papillary muscle outward displacement into the mediolateral (commissure-commissure) direction. We previously proposed the idea of a mediolateral papillary separation effect on mitral geometry (Figure 1, $D-F) .{ }^{5}$ Mediolateral displacement of anterior and posterior papillary muscles lifts up the central portion of the anterior and posterior leaflets and reinforces leaflet tethering (Figure 1, $F)$. In contrast, apical displacement of both papillary muscles does not 
lift up the central portion of both leaflets and results in less tethering of leaflets (Figure 1, E). With 3-dimensional analysis by means of multislice computed tomography, we have demonstrated that the IPMD, which reflects the degree of papillary separation, is a strong determinant of severity of FMR. Moreover, we found that when the papillary muscle tethering distance was resolved into apical and nonapical (posteromedial or posteromedial) components, the severity of FMR was strongly correlated with nonapical components. These findings strongly support our concept of papillary separation.

Another important concept is that valvular tenting of mitral leaflets is determined by the positions of the anterior and posterior papillary muscles. Leaflet tethering is geometrically restricted by the length of the main and aligning chords. Excessive mediolateral displacement of unilateral papillary muscle therefore lifts not only the ipsilateral portion of mitral leaflets but also the contralateral portion in cooperation with contralateral papillary muscle. ${ }^{6}$ This could be a possible explanation why the IPMD is strongly correlated with the severity of FMR.

In summary, apical displacement of papillary muscles has less effect on leaflet tethering and severity of FMR than does posteromedial or posterolateral displacement. Papillary separation results from nonapical outward displacement of papillary muscle, lifting up central portions of both mitral leaflets, which leads to insufficient mitral closure. Leaflet tethering of both mitral leaflets is determined by coordination of the anterior and posterior papillary muscles. The IPMD, reflecting the degree of papillary separation and bilateral papillary muscle displacement, could therefore be a plausible dominant determinant of FMR in patients with left ventricular dysfunction.

Shuichiro Kaji, MD
Kitae Kim, $M D$

Yutaka Furukawa, MD

Department of Cardiovascular

Medicine

Kobe City Medical Center General

Hospital

Kobe, Japan

\section{References}

1. Otsuji Y, Handschumacher MD, Schwammenthal E, Jiang L, Song JK, Guerrero JL, et al. Insights from three-dimensional echocardiography into the mechanism of functional mitral regurgitation: direct in vivo demonstration of altered leaflet tethering geometry. Circulation. 1997;96:1999-2008.

2. Kumanohoso T, Otsuji Y, Yoshifuku S, Matsukida K, Koriyama C, Kisanuki A, et al. Mechanism of higher incidence of ischemic mitral regurgitation in patients with inferior myocardial infarction: quantitative analysis of left ventricular and mitral valve geometry in 103 patients with prior myocardial infarction. J Thorac Cardiovasc Surg. 2003;125:135-43.

3. Menicanti L, Di Donato M, Frigiola A, Buckberg G, Santambrogio C, Ranucci M, et al. Ischemic mitral regurgitation: intraventricular papillary muscle imbrication without mitral ring during left ventricular restoration. J Thorac Cardiovasc Surg. 2002;123:1041-50.

4. Lomholt M, Nielsen SL, Hansen SB, Andersen NT, Hasenkam JM. Differential tension between secondary and primary mitral chordae in an acute invivo porcine model. J Heart Valve Dis. 2002;11: 337-45.

5. Kim K, Kaji S, An Y, Nishino T, Tani T, Kitai T, et al. Interpapillary muscle distance independently affects severity of functional mitral regurgitation in patients with systolic left ventricular dysfunction. J Thorac Cardiovasc Surg. 2014;148:434-40.e1.

6. Kim K, Kaji S, An Y, Yoshitani H, Takeuchi M, Levine RA, et al. Mechanism of asymmetric leaflet tethering in ischemic mitral regurgitation: $3 \mathrm{D}$ analysis with multislice CT. JACC Cardiovasc Imaging. 2012;5:230-2.

http://dx.doi.org/10.1016/ j.jtcvs.2014.09.072

\section{MANAGEMENT OF MILD AORTIC VALVE STENOSIS IN RHEUMATIC MITRAL SURGERY \\ To the Editor:}

We read with great interest the article by Hwang and colleagues ${ }^{1}$ on the management of mild aortic valve lesions during surgery for rheumatic mitral valve. We are grateful to them for their excellent and meaningful work, for we are facing the same dilemma occasionally.

In this study, Hwang and colleagues $^{1}$ demonstrated that mild rheumatic aortic stenosis frequently progresses in patients undergoing surgery for rheumatic mitral valve, regardless of initial treatment strategy. We agree with their conclusion. In our practice, the aortic valve should be always inspected in this scenario, especially in young patients. If the valve is found to be grossly deformed or with advanced commissural fusion, aortic valve replacement is performed at the same time. Very little information is available about progression of rheumatic aortic stenosis. Rates of progression of mild stenosis are neither uniform nor predictable. $^{2}$ Usually the progression of aortic stenosis is slow, but in certain patients it may be remarkably rapid. Progression of stenosis from the mild to the severe form has been seen in as little as 2 to 3 years. ${ }^{3}$ Otto and colleagues ${ }^{4}$ found that the presence of mild aortic stenosis signifies a greater degree of aortic valve disease than mild aortic regurgitation and that turbulence caused by a stenotic valve can contribute to leaflet damage and rapid progression of the lesion. We try to avoid a potential reoperation in the near future, which is frequently denied by patients' families because of the costs. We therefore would like to know whether Hwang and colleagues $^{1}$ inspected the stenotic aortic valve during the primary mitral operation.

Hwang and colleagues ${ }^{1}$ also found that in the 113 patients in the no treatment group who underwent postoperative echocardiography, the grade of the aortic valve lesion improved to less than mild degree and became aggravated to moderate degree in only 1 patient. We would like to know the diagnosis of the mitral valve disorder in the patient with aggravation. In the presence of severe mitral stenosis, the hemodynamic effects of aortic stenosis remain masked. We 